\title{
IEEE 802.16: WiMAX Overview, WiMAX Architecture
}

\author{
Mojtaba Seyedzadegan and Mohamed Othman
}

\begin{abstract}
WiMax, the Worldwide Interoperability for Microwave Access is a new technology dealing with provision of data over long distance using wireless communication method in many different ways. Based on IEEE 802.16 WiMax is claimed as an alternative broadband rather than cable and DSL. This paper is a quick technical overview and covers:WiMAX overview(Fundamental Concept; Technology; Standard update) andWiMAX architecture(Network and Node Architectures; Physical Layer; MAC Layer)
\end{abstract}

Index Terms - Broadband, IEEE 802.16, WiMAX.

\section{WIMAX OVERVIEW}

\section{A. Fundamental Concept}

WiMAX (Worldwide Interoperability for Microwave Access) is a connection-oriented wide area network [1]. It supports high bandwidth and hundreds of users per channel at speeds similar to currently seen for DSL, Cable or a T1 connection; Promises to provide a range of 30 miles as an alternative to wired broadband like cable and DSL. It could potentially provide broadband access to remote places. Use point-to-multipoint $(P 2 M P)$ architecture. It is designed for delivering broadband seamless quality multimedia services to the end users. "WiMAX combines the familiarity of Wi-Fi with the mobility of cellular that will deliver personal mobile broadband that moves with you" [2].

\section{B. WiMAX Devices}

- A WiMAX base-station which is similar to a cellular tower, except that it can cover of almost of 3,000 square miles ( 8,000 square $\mathrm{km})$.

- A WiMAX receiver could be standalone tower or a PCMCIA card inserted into your laptop. By having such large coverage areas, potential problems of handoffs associated with 802.11 seems to be solved.

\section{Challenge}

QoS is a challenge. Efficient scheduling design is left for designers and developers. Consequently providing QoS Scheduling Architecture for WiMAX Base Stations is a challenge for system developers.

\section{WiMAX and Wireless Mesh Network}

"If WiMAX and Wi-Fi technologies can work together in a mesh environment, it would bring at least a temporary resolution to the issue of whether or not the technologies are actually competitive with one another. It seems that as the

Manuscript received November 13, 2012; revised March 7, 2013.

The authors are with the Department of Communication Technology and Network, Faculty of Computer Science and Information Technology, Universiti Putra Malaysia, 43400 UPM, Serdang, Selangor D.E, Malaysia (email: mojtaba@fsktm.upm.edu.my,mothman@fsktm.upm.edu.my). wireless newcomer, WiMAX always has been viewed as being potentially competitive with every other wireless broadband access technology, including 3G and Wi-Fi, but using WiMAX as the backhaul method in a Wi-Fi mesh topology will prove the two technologies to be complementary.'[3].

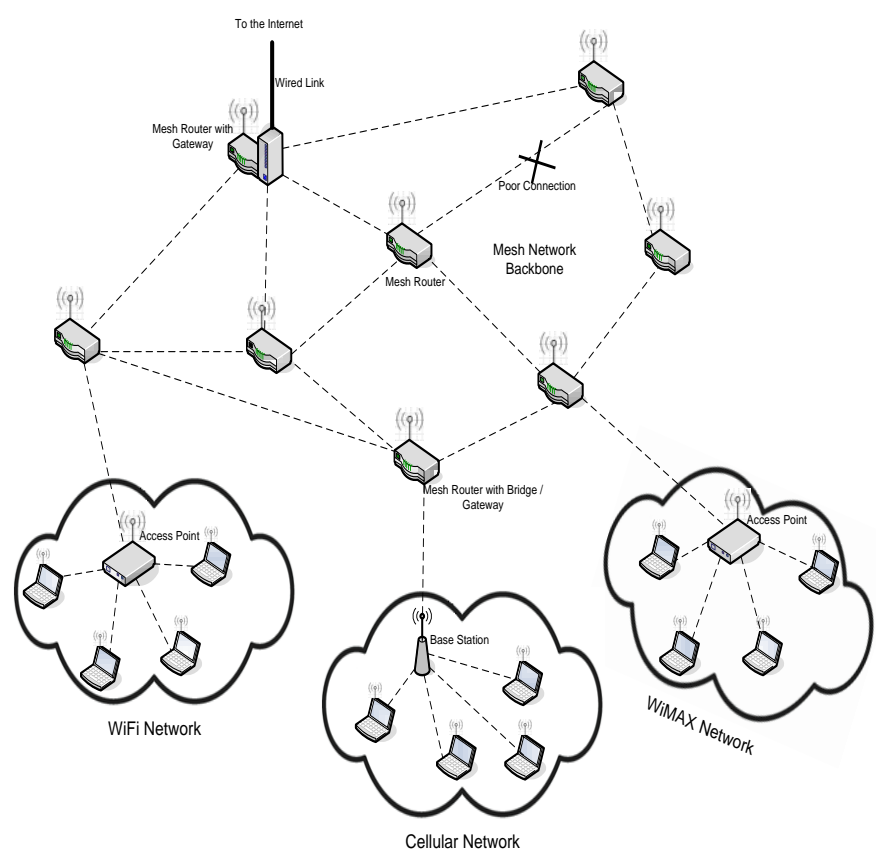

Fig. 1. Overview of a backbone mesh network and connections to WiFi, WiMAX, and wireless cellular networks [4]

\section{E. WiMAX vs. $3 G$}

The common Misconceptions about WiMAX \& 3G CDMA are [5]:

\section{1) Cost considerations}

- WiMAX networks cost less (by a factor of $2-10 x$ )

- WiMAX devices will cost less

- WiMAX IPR cost will be lower

- WiMAX has larger ecosystem (many participants, more competition)

2) Services and revenue

- WiMAX is optimized for data, 3G CDMA is for voice

- 16d WiMAX systems are easily upgraded to mobility

- WiMAX is Wi-Fi with greater range

\section{F. IEEE 802.16 Features}

802.16 is used for WiMAX with the features:

- Uses wireless link with microwave or millimeter waves.

- Uses licensed spectrum

- Provides public network service to fee-paying customers

- Uses point-to-multipoint $(P 2 M P)$ architecture

- Simple stationary rooftop or tower-mounted antennas

- Provide broadband and QoS guarantee data transmissions.

\section{G. IEEE 802.16 Standard History}




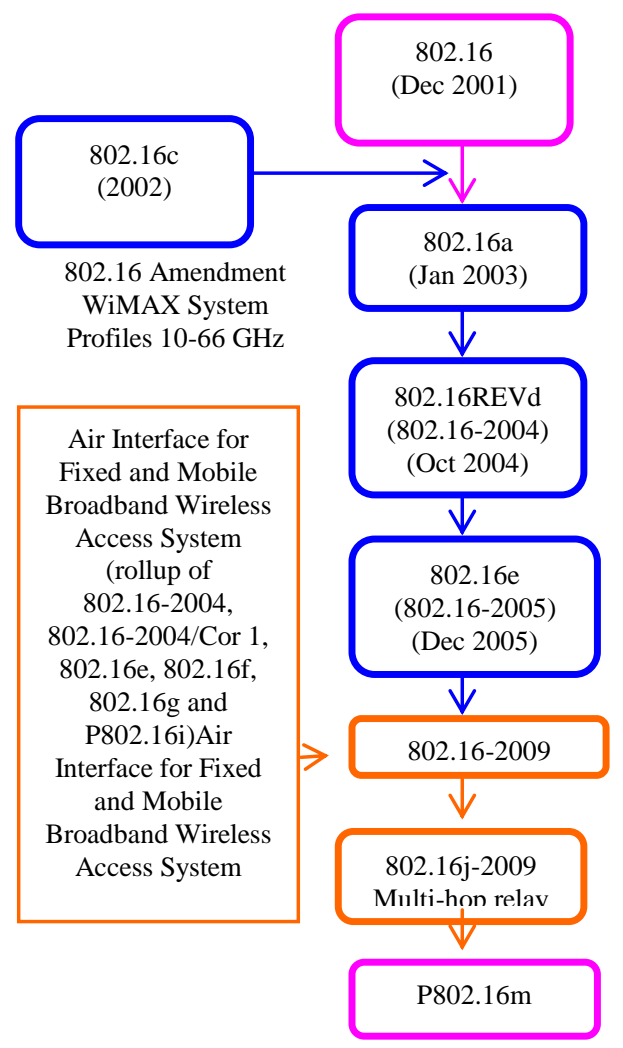

H. 802.16 Standards

\begin{tabular}{|c|c|c|c|c|}
\hline & IEEE 802.16 & IEEE 802.16a/ Rev'd & IEEE $802.16 \mathrm{e}$ & IEEE 802.16j- 2009 \\
\hline Completed & $\begin{array}{c}\text { December } \\
2001\end{array}$ & May 2004 & Mid-2005 & May 2009 \\
\hline Frequency & $10-66 \mathrm{GHz}$ & $2-11 \mathrm{GHz}$ & $2-6 \mathrm{GHz}$ & $\begin{array}{c}10-66 \mathrm{GHz} \\
\text { Below } 11 \mathrm{GHz}\end{array}$ \\
\hline Application & Backhaul & $\begin{array}{c}\text { Wireless DSL and } \\
\text { Backhaul }\end{array}$ & Mobile Internet & Wireless MAN \\
\hline $\begin{array}{l}\text { Channel } \\
\text { Conditions }\end{array}$ & $\begin{array}{l}\text { Line of Sight } \\
\text { Only }\end{array}$ & Non-Line of Sight & Non-Line of Sight & $\begin{array}{l}\text { LOS } \\
\text { NLOS }\end{array}$ \\
\hline Bit Rate & $\begin{array}{l}32-134 \mathrm{Mbps} \\
\text { at } 28 \mathrm{MHz} \\
\text { Channelization }\end{array}$ & $\begin{array}{l}\text { Up to } 75 \text { Mbps at } 20 \\
\mathrm{MHz} \text { Channelization }\end{array}$ & $\begin{array}{c}\text { Up to } 15 \mathrm{Mbps} \text { at } 5 \\
\mathrm{MHz} \\
\text { Channelization }\end{array}$ & $\begin{array}{l}\text { Up to } 120 \mathrm{Mbps} \text { at } \\
25 \mathrm{MHz} \text { or } 28 \mathrm{MHz} \\
\text { Channelization }\end{array}$ \\
\hline Modulation & $\begin{array}{l}\text { QPSK, 16QAM } \\
\text { and 64QAM }\end{array}$ & $\begin{array}{c}\text { OFDM } 256, \text { OFDMA } \\
2048 \\
\text { QPSK,16QAM,64QAM }\end{array}$ & $\begin{array}{l}\text { Same as } \\
\text { 802.16d,Scalable } \\
\text { OFDMA }\end{array}$ & $\begin{array}{l}\text { BPSK, QPSK, 16- } \\
\text { QAM, 64-QAM, } \\
\text { OFDM, OFDMA }\end{array}$ \\
\hline $\begin{array}{l}\text { Channel } \\
\text { Bandwidths }\end{array}$ & $\begin{array}{c}20,25 \text { and } 28 \\
\mathrm{MHz}\end{array}$ & $\begin{array}{c}\text { Selectable Channel } \\
\text { Bandwidths Between } \\
1.5 \text { and } 20 \mathrm{MHZ}\end{array}$ & Same as $802.16 \mathrm{~d}$ & $\begin{array}{c}25 \mathrm{MHz} \text { or } 28 \mathrm{MHz} \\
\text { are typical }\end{array}$ \\
\hline
\end{tabular}

\section{WIMAX ARCHITECTURE}

\section{A. WiMAX Network Architecture}

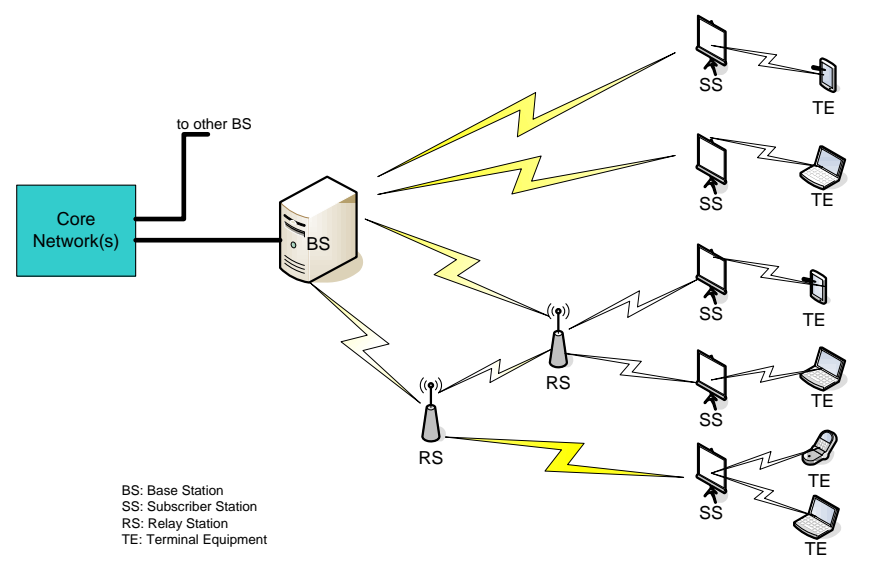

Fig. 2. IEEE 802.16 network architecture
WiMAX architecture consists of two types of fixed (non mobile) stations:

- Subscriber Stations (SS): serves a building (business or residence)

- Base station (BS): connects to public network and provide $\mathrm{SS}$ with first-mile access to public networks

The communication path between SS and BS has two directions:

- Uplink (from SS to BS)

- Downlink (from BS to SS)

\section{B. WiMAX Layer Architecture}

Physical layer functions are encoding/decoding of signals, preamble generation/removal, and bit transmission/reception.

In the Data link layer, medium access control functions are:

- On transmission, assemble data into a frame with address and error detection fields

- On reception, disassemble frame, and perform address recognition and error detection

- Govern access to the wireless transmission medium For the convergence layer, functions are:

Encapsulate PDU framing of upper layers into native 802.16 MAC/PHY frames, map upper layer's addresses into 802.16 addresses, translate upper layer QoS parameters into native 802.16 MAC format, and adapt time dependencies of upper layer traffic into equivalent MAC service.

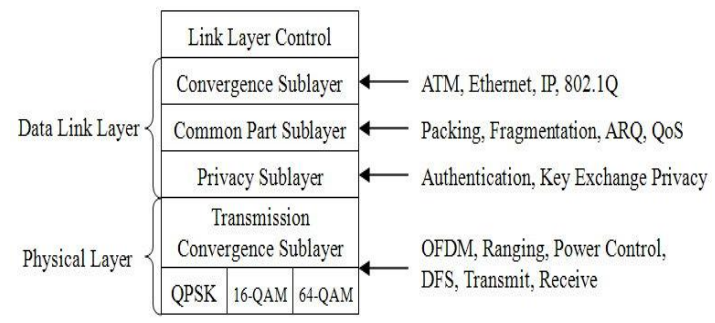

C. Channel Acquisition Process between an SS and BS [6]

BS

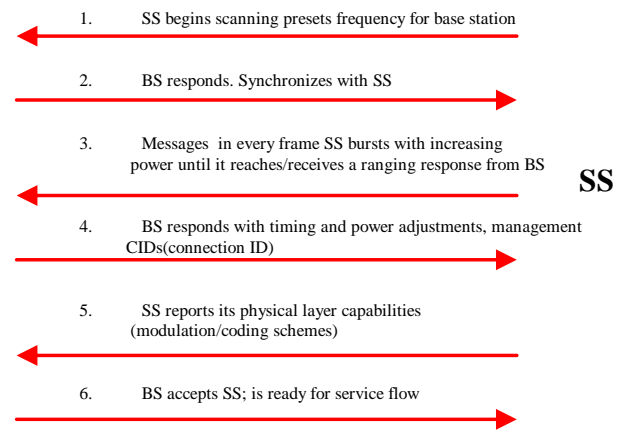

D. SS Authentication and Registration [5]

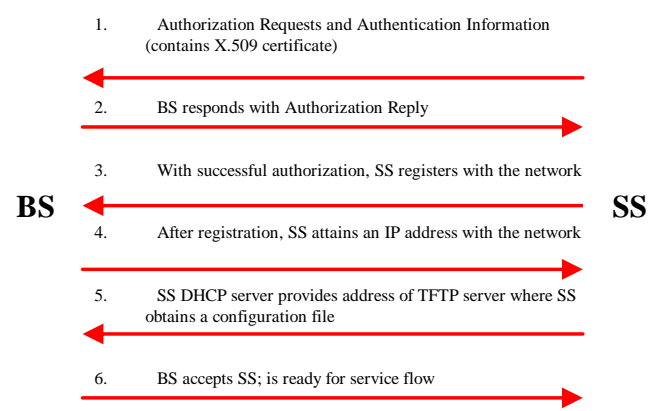




\section{E. Multimedia over WiMAX}

The basic idea of multimedia streaming is transporting different types of desired media content to the user over a network, and displaying it to the user. Both, 'Live and interactive audio/video' on the other hand, involve a client and two servers.

WiMAX has the capability to deliver broadband speeds over $70 \mathrm{Mb} / \mathrm{s}$ in an efficient manner. A sophisticated QoS scheme is needed to have a real time experience of voice and Video applications.

The architecture of WiMAX Base-Station with QoS provision for Multimedia Application is as follow:

Base Station (BS) QoS architecture:

- A compensation block receives Carrier to Interference Noise Reports (CINR), also bandwidth requests, from substations (SSs).

- Based on reports, compensation block labels a flow as a bad or a good flow.

- The Connection Identifiers (CIDs) belonging to a bad flow are marked as banned by the compensation block.

- The compensation block connects to an intelligent packet scheduler to select the appropriate flow for transmission. A system channel aware.

- Before transmitting a packet, the scheduler checks whether the packet has a banned CID to stop transmitting it.

- The classifier gets feedback from the compensation block.

- Connections with separate connection IDs (CIDs) and classifies traffic based on a class-based approach are differentiated.

- QoS admits frames with only those CIDs that can be serviced without any significant delay to the real time voice and video applications, thus:

- It rejects a CID that experiences bad channel quality and allows unmarked CIDs to utilize the channel at its maximum efficiency.

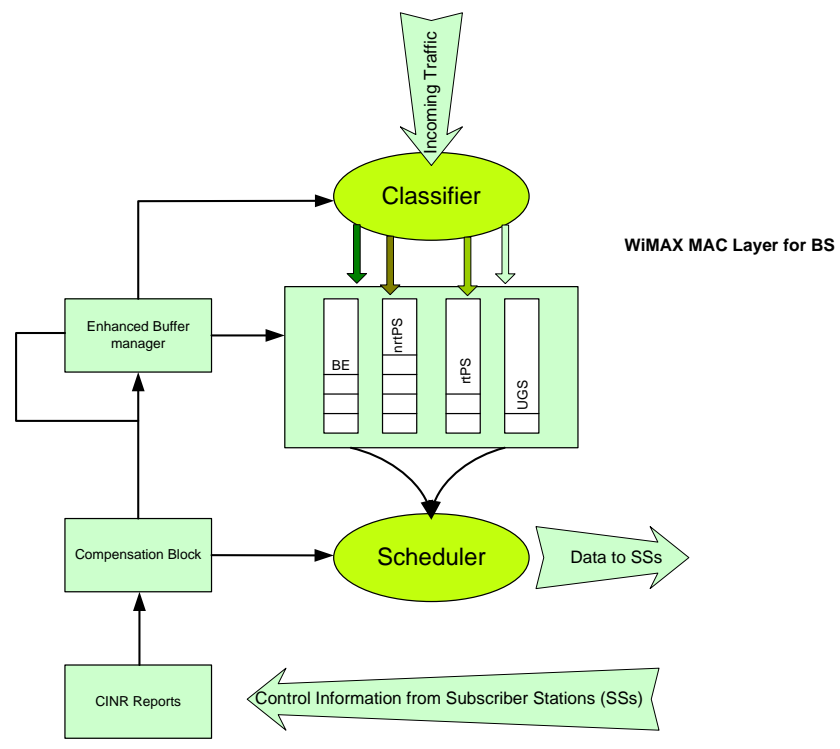

Fig. 3. WiMAX BS Architecture

\section{F. Physical Layer}

The physical layer supports:

- OFDM: Orthogonal Frequency Division Multiplexing

- TDD: Time Division Duplex

- FDD: Frequency Division Duplex

- QPSK: Quadrature Phase Shift Keying
G. MAC Layer

WiMAX MAC layer is a point to multipoint protocol (P2MP). It supports high bandwidth and hundreds of users per channel. It utilizes spectrum efficiently by supporting bursty traffic. The MAC convergence sub layer offers support for ATM, Ethernet, 802.1Q, IPv4, IPv6 (a possible future support for PPP, MPLS etc). The core MAC layer provides packet fragmentation, ARQ and QOS. The MAC Privacy Sub layer integrates security features in WiMAX. Authentication, encryption and Key exchange functionality are provided in MAC sub layer.

- Privacy Sublayer

1) Encapsulation protocol

- Encrypt or decrypt data

1) Privacy Key Management (PKM) protocol

- Secure distribution of keying data (BS to SS)

1) Security Association (SA)

- Identified by SAID

- Contains cryptographic suite and security info

\section{H. WiMAX PHY and MAC Summary}

\section{- PHY}

1) MIMO

2) Modulation

3) OFDM, Modulation technique, spread spectrum (used in e.g DSL, WLAN, WiMAX)

- MAC, control plane, user plane

1) Manage resources on the air interface

2) QoS for multimedia and video streaming

3) ARQ

4) Mobility Management

5) Radio Resource Management

6) Location

\section{CONCLUSION}

WiMAX Technology is facing many hurdles in market while it has some great advantages which make it a technology of today. This paper provided the quick and technical overview of concept, technology, standard, and architecture for IEEE 802.16 WiMAX.

\section{REFERENCES}

[1] IEEE Standard for Local and Metropolitan Area Networks, Part 16: Air Interface for Broadband Wireless Access Systems IEEE Std $802.16^{\mathrm{TM}}-2009$.

[2] Intel leap ahead. Welcome to Your Internet Future. Intel 2007. (August 30, 2010). [Online]. Available: http://download.intel.com/network/connectivity/products/wireless/wel come-to-your-internet-future. pdf

[3] Dan O'Shea. WiMAX Makes a Mesh. (Oct. 17, 2005). [Online]. Available:

http://connectedplanetonline.com/mag/telecom_wimax_makes_mesh/i ndex $2 . h t m l$

[4] N. F. Mir, Computer and Communication Networks, Printice Hall, 2006.

[5] WiMAX vs 3G CDMA. (January 2008). [Online]. Available: http://www.qualcomm.com.

[6] WiMAX Made Simple. How WiMAX Works. (December 15, 2009) [Online]. Available: http://wimax-made-simple.blogspot.com/2009/12/how-wimax-works. html 


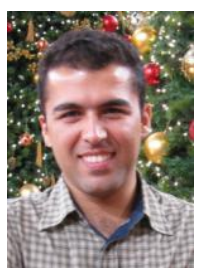

Mojtaba Seyedzadegan currently is with the Wireless and Mobile Networks Laboratory, Department of Communication Technology and Network, Faculty of Computer Science and Information Technology, Universiti Putra Malaysia (UPM). His field of study is

Computer Networks and his interests include Architectural Design of WiMAX and Wireless Mesh Networks, Fairness in Wireless LANs, Network Management, and Graph Theory.

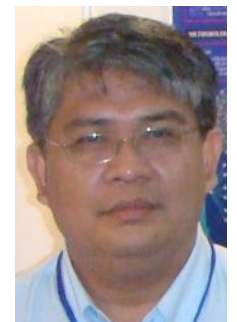

Mohamed Othman is a professor of computer science in the Department of Communication Technology and Network, Universiti Putra Malaysia (UPM), since 2001. In April 2008, he was appointed as a deputy director of InfoComm Development Centre (iDEC) at the same university. He received his $\mathrm{PhD}$ in Computer Science from the Department of Industrial Computing, Universiti Kebangsaan Malaysia with distinction (Best PhD Thesis in 2000 awarded by Sime Darby Malaysia and Malaysian Mathematical Science Society) in 1999. Since 1989, in total, he had published more than 200 journal papers and more than 300 conference papers, both at national and international levels. He has expertise in several areas of computer science such as paralle and distributed algorithms, grid computing, high-speed computer network and scientific computing. 